\title{
Sperm proteasome degrades egg envelope glycoprotein ZP1 during fertilization of Japanese quail (Coturnix japonica)
}

\author{
Tomohiro Sasanami $^{1}$, Kenichi Sugiura ${ }^{1}$, Toshinobu Tokumoto ${ }^{2}$, Norio Yoshizaki $^{3}$, Hideo Dohra ${ }^{4}$, \\ Shunsuke Nishio ${ }^{6}$, Shusei Mizushima ${ }^{1}$, Gen Hiyama ${ }^{5}$ and Tsukasa Matsuda ${ }^{6}$ \\ Departments of ${ }^{1}$ Applied Biological Chemistry, Faculty of Agriculture and ${ }^{2}$ Biology, Faculty of Science, Shizuoka \\ University, 836 Ohya, Shizuoka 422-8529, Japan, ${ }^{3}$ Department of Agricultural Science, Gifu University, \\ Gifu 501-1193, Japan, ${ }^{4}$ Institute for Genetic Research and Biotechnology, Shizuoka University, 836 Ohya, \\ Shizuoka 422-8529, Japan, ${ }^{5}$ Department of Animal Science, McGill University, Lakeshore Road, \\ Ste-Anne-De-Bellevue, Quebec, Canada, H9X 3 V9 and ${ }^{6}$ Graduate School of Bioagricultural Sciences, \\ Nagoya University, Furo-cho, Chikusa-ku, Nagoya 464-8601, Japan \\ Correspondence should be addressed to T Sasanami; Email: atsasan@ipc.shizuoka.ac.jp
}

\begin{abstract}
At the time of fertilization, the extracellular matrix surrounding avian oocytes, termed the perivitelline membrane (pvm), is hydrolyzed by a sperm-borne protease, although the actual protease that is responsible for the digestion of the pvm remains to be identified. Here, we show evidence that the ubiquitin-proteasome system is functional in the fertilization of Japanese quail. The activities for the induction of the acrosome reaction and binding to ZP3 as revealed by ligand blotting of purified serum ZP1 are similar to those of pvm ZP1. Western blot analysis of purified ZP1 and ZP3 by the use of the anti-ubiquitin antibody showed that only pvm ZP1 was reactive to the antibody. In vitro penetration assay of the sperm on the pvm indicated that fragments of ZP1 and intact ZP3 were released from the pvm. Western blot analysis using the anti-20S proteasome antibody and ultrastructural analysis showed that immunoreactive proteasome was localized in the acrosomal region of the sperm. Inclusion of specific proteasome inhibitor MG132 in the incubation mixture, or depletion of extracellular ATP by the addition of apyrase, efficiently suppressed the sperm perforation of the pvm. These results demonstrate for the first time that the sperm proteasome is important for fertilization in birds and that the extracellular ubiquitination of ZP1 might occur during its transport via blood circulation.

Reproduction (2012) $\mathbf{1 4 4} 423-431$
\end{abstract}

\section{Introduction}

In fertilization, the male and female gamete, sperm, and oocyte, unite to produce a zygote, a fertilized egg. Fertilization is achieved with multiple steps under precise and ingenious regulation. These steps include sperm chemotaxis/activation, sperm-egg binding, induction of the acrosome reaction (AR) of sperm, and membrane fusion of the gametes (Florman \& Ducibella 2006). When a sperm encounters the oocyte, it must pass through the extracellular coat overlaid on the surface of the oolemma, which is called the zona pellucida (ZP) in mammals and the vitelline envelope in marine invertebrates or in amphibians. It is believed that the spermborne protease hydrolyzes the egg extracellular coat and that the hole produced by the protease is the path for sperm penetration (Florman \& Ducibella 2006). It had long been believed that, in mammalian species, the sperm acrosin, a non-ATP-dependent serine protease localized in the sperm head, is indispensable for penetration of the sperm (McRorie \& Williams 1974). However, Baba et al. (1994) demonstrated that acrosinnull male mice produce sperm normal in motility and are fertile; therefore, acrosin is not essential for fertilization, at least in mouse. At present, acrosin can be interpreted as being responsible for the dispersal of the acrosomal contents in the AR (Yamagata et al. 1998). Sawada et al. reported that in marine invertebrate such as ascidians and sea urchins, sperm proteasomes is responsible for sperm penetration of the vitelline envelope, and the proteasome is a lytic agent called lysin, which is essential for disintegration of the egg extracellular matrix (Sawada et al. 2002a, Sakai et al. 2003, Yokota \& Sawada 2007a). A similar conclusion was drawn from mammalian fertilization, including that in mice, pigs, and man (Pasten et al. 2005, Kong et al. 2009, Zimmerman et al. 2011).

In avian species, the perivitelline membrane (pvm), which is the egg envelope homologous to ZP in 
mammals, is observed in follicles between granulosa cells and the ovum before ovulation (Wyburn et al. 1965). Fertilization occurs within the infundibular portion of the oviduct, and only the pvm encloses the oocyte at the time of fertilization. Sperm-egg interaction in avian species can be measured in vitro as the ability of the sperm to hydrolyze a hole in the pvm (Robertson et al. 2000). This hole is relatively large $(\sim 9-20 \mu \mathrm{m}$ in diameter) in comparison with the outer diameter of the sperm head $(0.6 \mu \mathrm{m})$ and can clearly be observed in a light microscope at low magnification. Using this in vitro assay, we demonstrated that an $\mathrm{N}$-glycan present on ZP1, one of the major components of pvm that is produced in the liver under estrogen control (Sasanami et al. 2003), has the ability to induce AR in Japanese quail (Sasanami et al. 2007). More recently, we found that the $45 \mathrm{kDa}$ sperm acrosin localized on the sperm plasma membrane is responsible for binding the sperm to the pvm of the oocyte but is not important for digestion of the pvm glycoproteins (Sasanami et al. 2011). These results indicate that the actual lysin that functions during avian fertilization is not acrosin but another protease existing in the sperm. Additionally, we found that ZP1, which circulates in the bloodstream, received some modifications during transport to the pvm. It is from the fact that when ZP1 purified from the serum of laying quail was injected into different birds, the signal of exogenous ZP1 was detected in the pvm, whereas ZP1 derived from the pvm failed to incorporate into the pvm (Kinoshita et al. 2008). In this report, we demonstrate for the first time that the ubiquitin-proteasome system functions in avian fertilization. Moreover, we also show that the target protein of sperm proteasome is ZP1 in quail and suggest that the ubiquitination of ZP1 might take place during the transport from the bloodstream to the pvm matrix.

\section{Results \\ Differences in biological activities and biochemical properties between serum and pvm ZP1s}

In order to differentiate the biological activities and biochemical properties of purified serum and pvm ZP1, we compared the potential of the protein to induce an AR of ejaculated sperm. As shown in Fig. 1A, serum and pvm ZP1 possess similar activities for the induction of acrosomal exocytosis. In addition, when the purified proteins were incubated with $\left[{ }^{3} \mathrm{H}\right]$-labeled ZP3 to compare its binding to the ZP3, their abilities to interact with ZP3 did not differ markedly from each other (Fig. 1B). The apparent molecular weight as determined by SDS-PAGE as well as the reactivities of the proteins to our anti-ZP1 antiserum also did not differ much, but we found that only the pvm ZP1 reacted well to the anti-Ub antibody (Fig. 2). This immunoreaction is specific because the serum ZP1 did not react with the anti-Ub antibody and nonspecific mouse IgG failed to react with
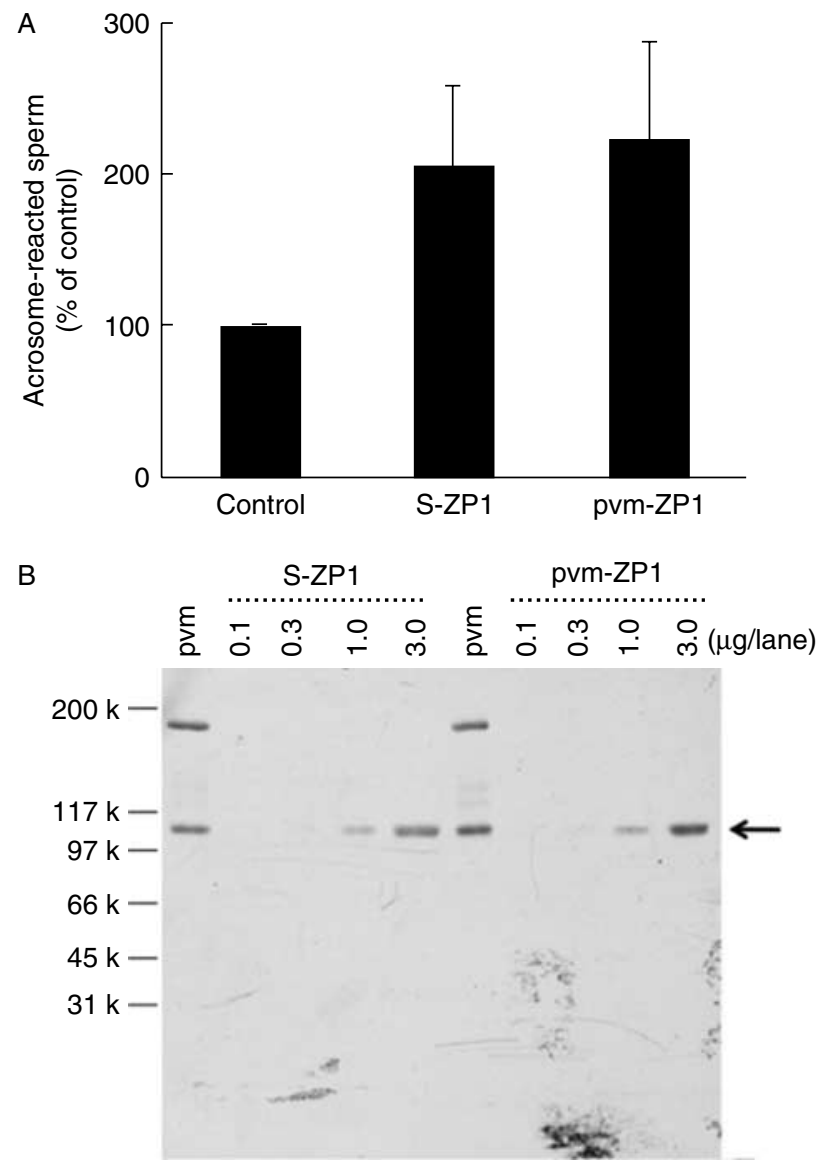

Figure 1 Comparison of biological activity of serum and pvm ZP1. (A) The ejaculated sperm were incubated in the presence or absence of purified ZP1 at $39{ }^{\circ} \mathrm{C}$ for $30 \mathrm{~min}$. After incubation, the percentage of the acrosome-reacted sperm was calculated as described in the Experimental procedures. Values are mean \pm s.D. of three independent experiments. (B) Ligand blot analysis of purified ZP1 with $\left[{ }^{3} \mathrm{H}\right]$-labeled ZP3. Purified serum (S-ZP1) or pvm (pvm-ZP1) ZP1 were separated by SDS-PAGE and then electrotransferred onto a PVDF membrane. Binding of $\left[{ }^{3} \mathrm{H}\right]$-labeled ZP3 to ZP1 was detected by ligand blot analysis. As a positive control, pvm lysate (10 $\mu \mathrm{g} / \mathrm{lane})$ was subjected to SDS-PAGE and detected in the same manner. Representative results of repeated experiments are shown. An arrow indicates the radiolabeled signal of monomeric ZP1.

the purified ZP1s. On the other hand, the purified ZP3 treated with similar conditions did not react with the anti-Ub antibody.

\section{Degradation of ZP1 by ejaculated sperm}

The fact that pvm ZP1 is ubiquitinated led us to hypothesize that the ubiquitinated pvm ZP1 might be degraded by sperm-derived proteasome during fertilization. To confirm this hypothesis, we incubated the isolated pvm with ejaculated sperm, and the solubilized materials released from the pvm during incubation were analyzed by western blotting. As shown in Fig. 3A, the 30-min incubation with the sperm successively released 


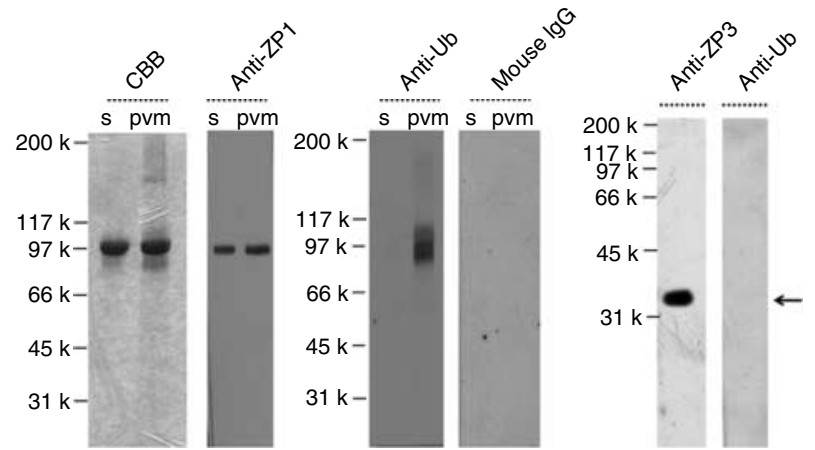

Figure 2 Detection of ubiquitination of pvm ZP1. Purified serum (S; $5 \mu \mathrm{g} /$ lane) or pvm ZP1 (pvm; $5 \mu \mathrm{g} /$ lane) was separated by SDS-PAGE and the protein was visualized by CBB staining. The same sample $(1 \mu \mathrm{g} /$ lane) was detected with western blotting using anti-ZP1 antiserum, anti-ubiquitin antibody, or normal mouse IgG. As shown in the right panel, purified ZP3 ( $1 \mu \mathrm{g} / \mathrm{lane})$ was separated with SDS-PAGE and was detected using anti-ZP3 antiserum or anti-ubiquitin antibody. Note that the immunoreactive band against the anti-ubiquitin antibody was seen in the lane of purified pvm ZP1 but not in the lane of the serum ZP1 or purified ZP3. An arrow indicates the band of ZP3. Results shown in the figure are representative of repeated experiments.

the fragments, which reacted with anti-ZP1 antiserum. These fragments are thought to be derived from pvm during incubation with the sperm because no band was seen in the absence of either sperm or pvm in the reaction mixture. When the same sample was detected with the anti-ZP3 antibody, immunoreactive $35 \mathrm{kDa}$ ZP3 was detected when the pvm and sperm were co-incubated. However, it should be noted that only intact $35 \mathrm{kDa}$ ZP3 was detected in the sample. These results indicate that the fragment of the ZP1 as well as intact ZP3 is released from the pvm during co-incubation with the sperm and that this phenomenon might be related to the hole formation during quail fertilization. In the next experiments, we compared the susceptibility of the serum and pvm ZP1 to break down by the sperm. As shown in Fig. $3 \mathrm{~B}$, the intensity of the $97 \mathrm{kDa}$ band of purified pvm ZP1 progressively decreased during incubation and completely disappeared after $60 \mathrm{~min}$ of incubation. However, the band of serum ZP1 remained after 60-min exposure to the sperm.

\section{Detection of proteasome in quail sperm}

In the next set of experiments, we tested whether a proteasome exists in the quail spermatozoa. To achieve this goal, we detected the sperm extracts by western blotting using anti-20S proteasome antibodies (Fig. 4). The membrane fraction prepared from the cavitated sperm by ultracentrifugation was shown to contain immunoreactive materials that reacted with anti- $\alpha 5$ (arrow in panel $A$, group -, lane 1), anti- $\alpha 6$ (arrow in panel $\mathrm{B}$, group -, lane 1), and anti- $\alpha 7$ antibodies (arrow in panel $\mathrm{C}$, group -, lane 1). The cytosol fraction also included the immunoreactive protein that appears at the same location in SDS-PAGE (lane 2 of group - in each panel). When each antibody was pre-absorbed by its respective antigen, the bands migrating to around $30 \mathrm{kDa}$ in both the membrane and the cytosol disappeared (lanes 1 and 2 of group + in each panel). These results suggest that the $30 \mathrm{kDa}$ bands reacting with anti- $\alpha 5$, anti- $\alpha 6$, or anti- $\alpha 7$ antibodies are authentic subunits of the proteasome in quail spermatozoa.
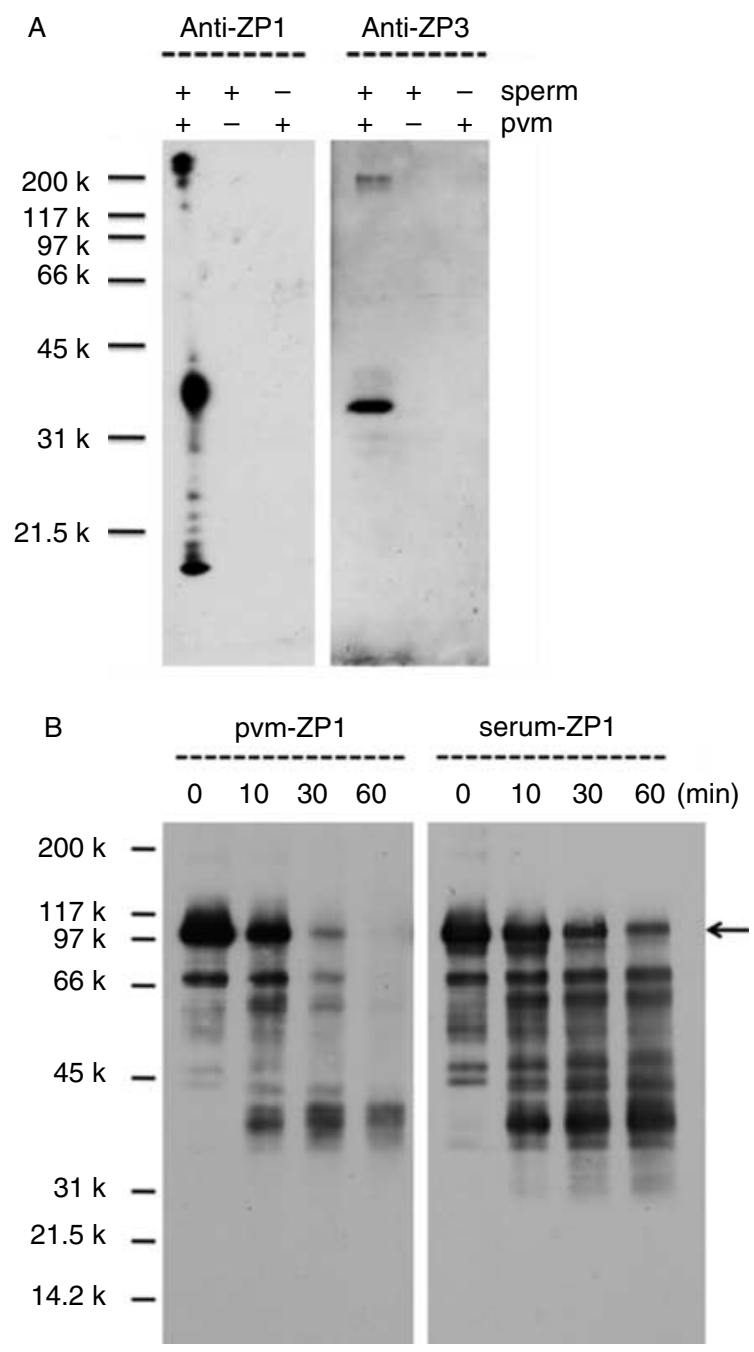

Figure 3 Degradation of ZP1 by ejaculated spermatozoa. (A) The pvm isolated from the largest follicles was incubated with $(+$ pvm and + sperm) or without (+ pvm and - sperm) ejaculated spermatozoa at $39^{\circ} \mathrm{C}$ for $30 \mathrm{~min}$. For the negative control, only sperm were incubated in the same conditions ( - pvm and + sperm). After the incubation, the degraded materials of the pvm were collected by centrifugation and detected with western blotting using anti-ZP1 or anti-ZP3 antiserum. Shown are representative results of repeated experiments. (B) $5 \mu \mathrm{g}$ of purified pvm ZP1 or serum ZP1 was incubated with ejaculated spermatozoa for $0,10,30$, or $60 \mathrm{~min}$ at $39^{\circ} \mathrm{C}$. After the incubation, the reaction mixtures were centrifuged and the supernatants detected using anti-ZP1 antiserum. An arrow indicates intact ZP1. Representative results of repeated experiments are shown. 
A

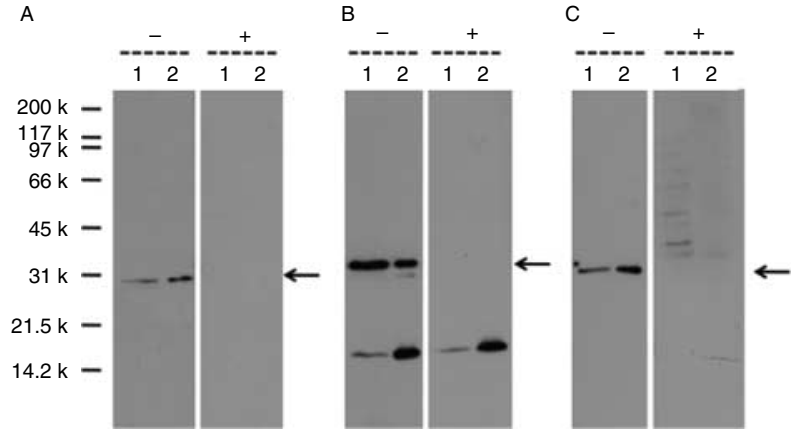

Figure 4 Detection of proteasome in the ejaculated sperm. The ejaculated sperm suspended in the buffer were cavitated as described in the Experimental procedures. After sedimentation of the debris by centrifugation, the supernatants was ultracentrifuged to separate the membrane (lane 1) and cytosol fractions (lane 2). Samples were then separated by SDS-PAGE and detected using anti-20S proteasome $\alpha 5$ subunit antiserum (group - in panel A), anti-20S proteasome $\alpha 6$ subunit antiserum (group - in panel B), or anti-20S proteasome $\alpha 7$ subunit antiserum (group - in panel C). Each antiserum was pre-absorbed with its respective antigen for $1 \mathrm{~h}$ at $4{ }^{\circ} \mathrm{C}$ to prepare control antiserum (group + in panels A, B, and C). Arrows indicate the band of proteasome.

Because the bands migrating around $17 \mathrm{kDa}$ that reacted with anti- $\alpha 6$ were not reduced by antigen absorption (panel B, group + ), this protein appears to react nonspecifically with the antibody. To analyze more precisely the localization of the proteasome, we performed immunoelectron microscopy for the detection of the antigen in ultra-thin sections of the sperm (Fig. 5). The immunogold particles were distributed in the acrosomal region of the sperm head as well as in the nucleus when the specimens were incubated with anti$\alpha 5$ antibody (panel A). No such accumulation of gold particles in the acrosome region was observed when the specimens were incubated with anti- $\alpha 5$ antibody that had been pre-absorbed with the antigen protein, but the signals persisted in the nucleus of the sperm (panel B). The immunogold accumulation in the nucleus was found to be a nonspecific signal due to the reactivities of the secondary antibody used because this signal was also found when the first antibody had been omitted (data not shown). These observations demonstrated that the antigen reactive to the anti- $\alpha 5$ antibody localizes in the acrosome region of the sperm head. Similar results were obtained when the specimens were reacted with anti- $\alpha 7$ antibody (data not shown).

\section{Effects of proteasome inhibitor and apyrase on the hole formation on the pvm by the sperm}

To provide further support to the idea that sperm proteasome is crucial for degrading the pvm by sperm, we examined whether MG132, a proteasome inhibitor, and apyrase, which catalyzes the hydrolysis of ATP and ADP, inhibits the in vitro perforation of the pvm by the sperm. When the pvm was incubated with the sperm in the presence of MG132, only few perforations were observed, whereas many holes were observed in the case of the vehicle control as well as the pvm that had been incubated in the presence of $\mathrm{E} 64 \mathrm{~d}$, a cysteine protease inhibitor (Fig. 6). In addition to these inhibitory effects on hole formation, apyrase also potently inhibited sperm perforation in vitro (Fig. 7). These results strongly suggest that sperm proteasome plays a crucial role in the degradation of the pvm during fertilization.
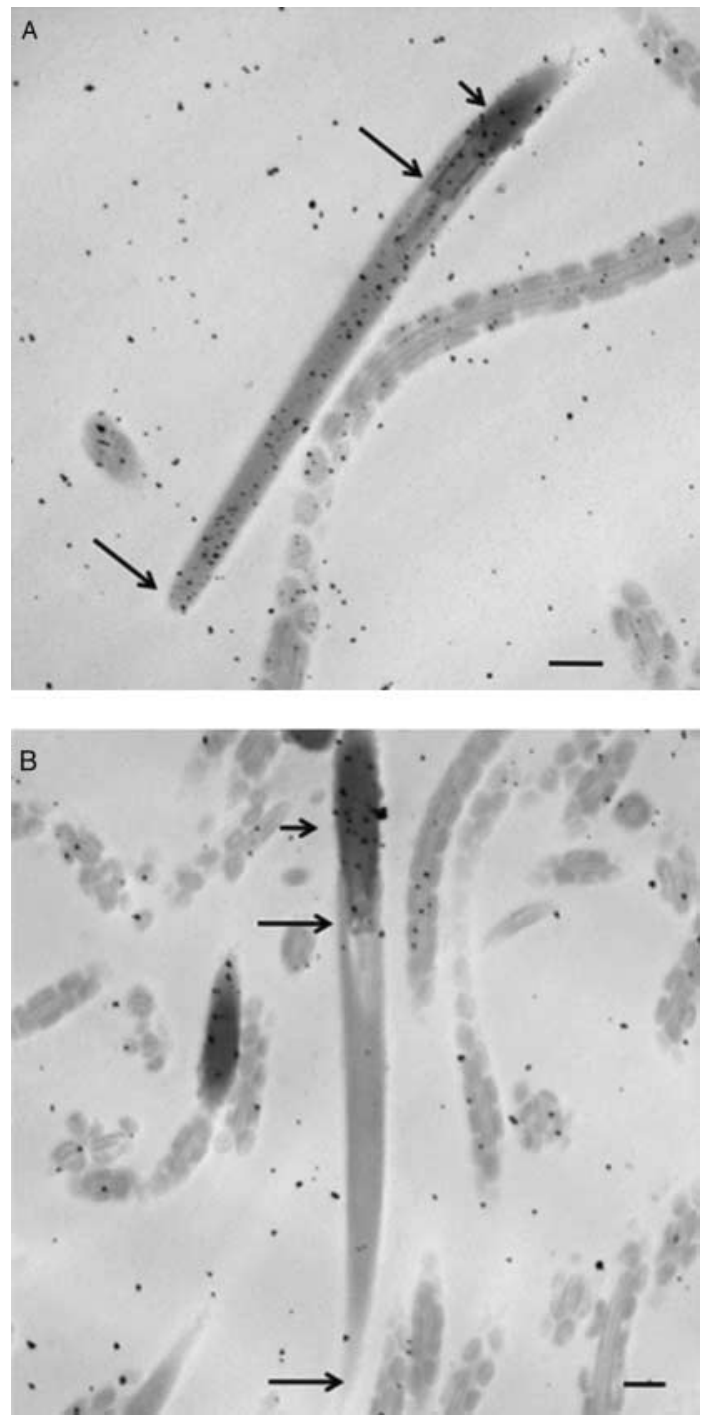

Figure 5 Immunoelectron microscopy of ejaculated sperm. Ultra-thin sections of quail sperm were incubated with the anti-20S proteasome $\alpha 5$ subunit antiserum (A) or normal guinea pig serum (B). The accumulation of gold particles was frequently found in the sperm acrosome (the acrosome located between the long arrows) when the sections were incubated with antiserum, whereas no accumulation was seen on the sections incubated with the antiserum, preincubated with antigen. Note that the immunogold particles were localized in both sections of the nucleus (short arrow) and that this signal was thought to be nonspecific. The representative results of repeated experiments are shown. Bar represents $500 \mathrm{~nm}$. 

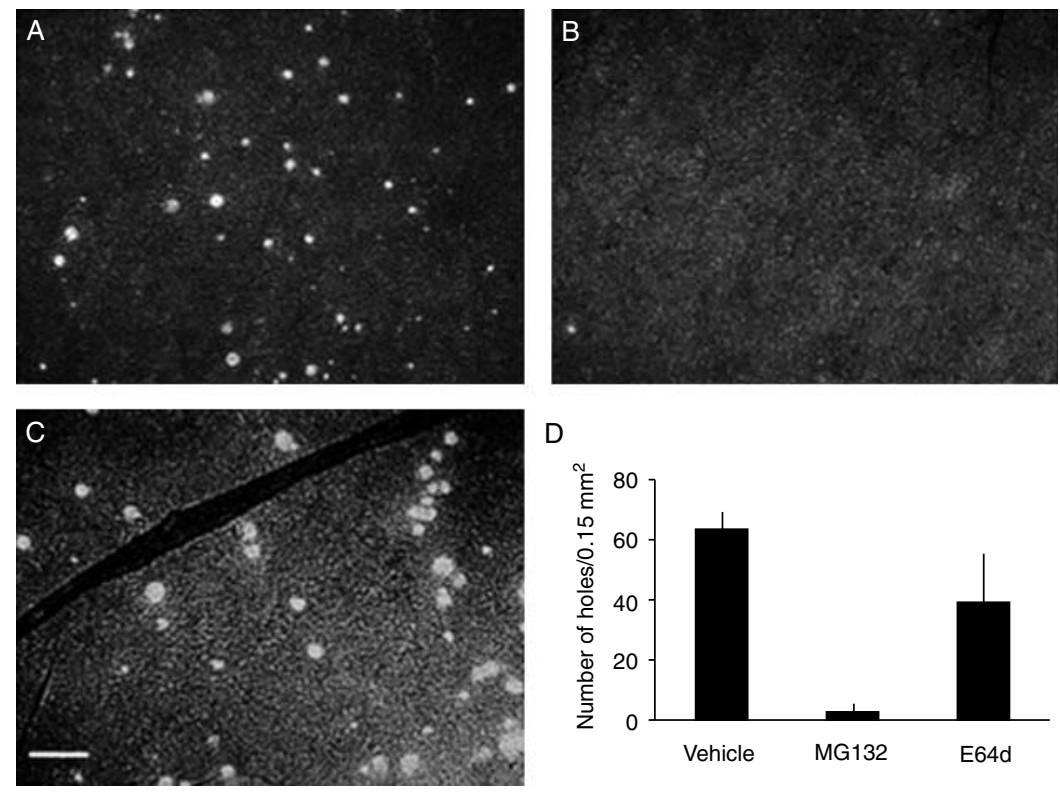

Figure 6 Effects of proteasome inhibitor on in vitro sperm perforation. The pvm isolated from the largest follicles was incubated with ejaculated sperm in the presence of dimethyl sulfoxide (A), $50 \mu \mathrm{M}$ MG132,

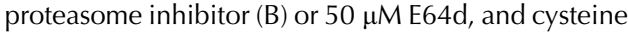
protease inhibitor (C) for $10 \mathrm{~min}$. After the incubation, the pvm was spread onto a glass slide and observed in a light microscope. (D) The number of holes per $0.15 \mathrm{~mm}^{2}$ was calculated from Image J Software and is expressed as mean \pm s.D. The images shown in (A), (B) and (C) are representative of repeated experiments. The values depicted in (D) and (E) were obtained from three independent experiments. Bar represents $50 \mu \mathrm{m}$.

\section{Discussion}

In this study, we demonstrated for the first time that the degradation of the pvm by sperm to produce holes in it during avian fertilization is mediated, at least in part, by the ubiquitin-proteasome system. This is strongly supported by the following findings. First, the ejaculated spermatozoa contain proteasome and that this proteasome is localized in the acrosomal region of the sperm head. Secondly, ZP1, a major constituent of the pvm, is reactive to anti-ubiquitin antibody, and the ejaculated sperm possess the potential to break down ZP1. However, the sperm fail to decompose ZP3, another major component of pvm, which appear not to undergo ubiquitin modification. Thirdly, the degradation of serum ZP1 that does not carry the ubiquitin is retarded compared with that of pvm ZP1. Fourthly, sperm protease activity to degrade the pvm is strongly disturbed by MG132, a potent proteasome inhibitor, but is not affected by E64d, a cysteine protease inhibitor. Fifthly, when the extracellular ATP is depleted by the addition of apyrase to the incubation mixture, hole formation is rarely observed. As described here, many lines of evidence obtained in this study suggest that the ubiquitin-proteasome system is pivotal to making holes in the pvm and subsequent sperm penetration in quail fertilization. It was reported in the chicken that acrosin, an acrosomal serine protease, plays an important role in fertilization and is believed to be involved in hole formation on the pvm during fertilization (Richardson et al. 1992, Slowinska et al. 2010). In our previous study, we found that sperm lysates have the potential to degrade the gelatin using zymography (Sasanami et al. 2011). However, anti-acrosin MAB failed to inhibit protease activity, whereas this antibody effectively disturbed the binding of the sperm to the pvm, indicating that acrosin plays a role in sperm-egg recognition in quail fertilization (Sasanami et al. 2011). In addition, the inhibition of the sperm penetration by apyrase also suggested that an ATP-dependent protease like proteasome, but not acrosin, participates in the process of the hydrolysis of the pvm.

In this study, we found that sperm degraded ZP1, but not ZP3, in the in vitro penetration assay and that this result demonstrates that the target for the sperm proteasome is ZP1 in avian species. It should be noted that the immunoreactive signal of the intact $35 \mathrm{kDa} Z \mathrm{ZP} 3$ was detected in the sample, indicating that ZP3 was also released from the pvm but failed to be degraded. Although the target site of the sperm proteasome for ZP1 is currently unknown, we previously reported that the binding of ZP1 and ZP3 for egg envelope formation is mediated by the ZP domain, a hallmark structure of ZP protein. We think that ZP3 might be released from the pvm as the ZP1-ZP3 complex.

The timing of the ubiquitination of the $\mathrm{ZP}$ proteins differs in different animals. For example, Hrc70, a sperm receptor in Halocynthia roretzi, was found to be ubiquitinated extracellularly by sperm-derived ubiquitination enzymes at the time of fertilization, and the ubiquitinated HRC70 protein is degraded with spermborne proteasome (Sawada et al. 2002a). In contrast, mammalian ZP protein, including that in mice, was ubiquitinated during the process of biosynthesis, and the intracellular ubiquitination of the ZP protein has been proposed (Sutovsky et al. 2004). Similar intracellular ubiquitination of the egg coat protein was observed in sea urchins (Yokota \& Sawada 2007a). The site of ubiquitination of ZP1 in quail is unclear. However, ZP1 might be modified extracellularly during transport in the bloodstream to the pvm. In electron microscopic 
analysis, a coarse cellular junction between the granulosa cells has been observed (Bellairs 1965), and very low-density lipoprotein and vitellogenin, a precursor protein of egg yolk, pass through these gaps
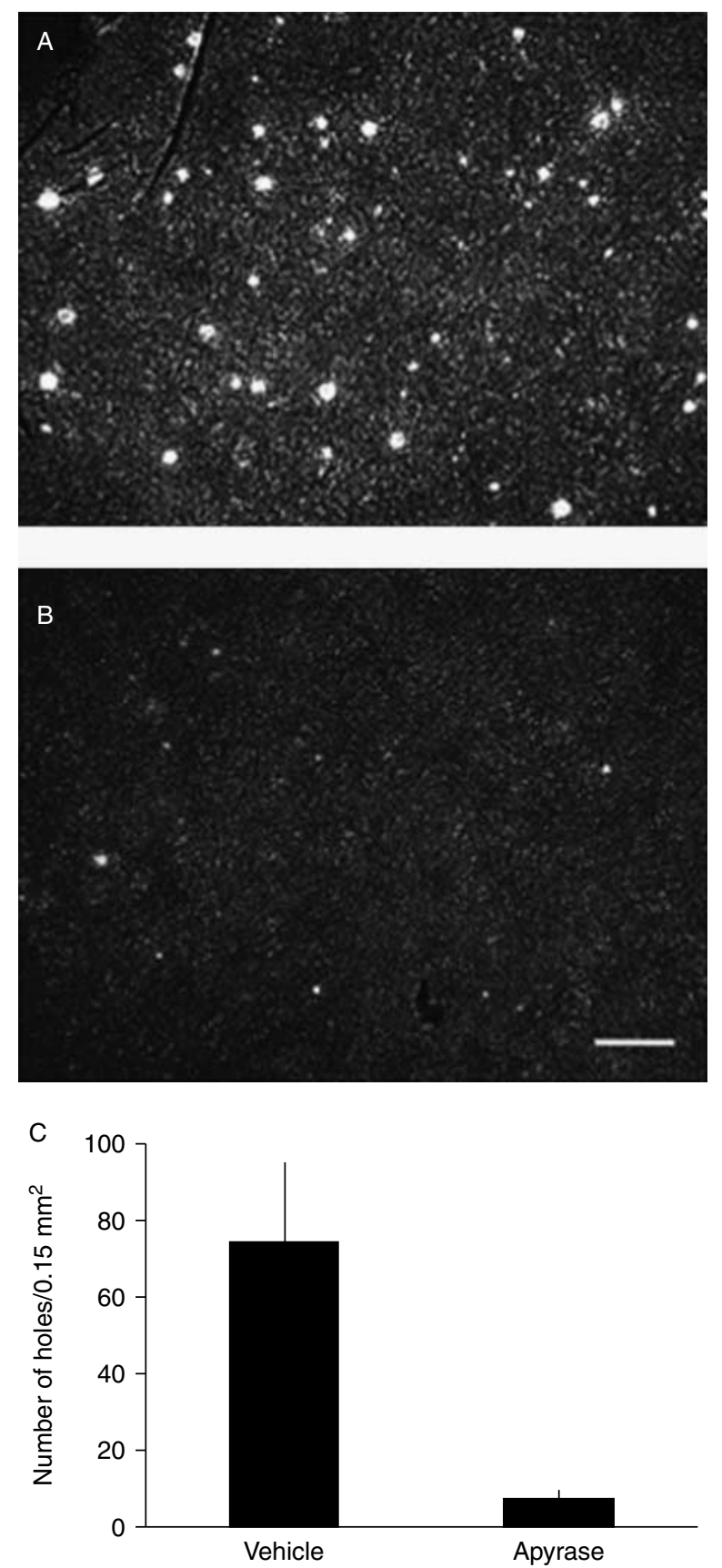

Figure 7 Apyrase inhibits in vitro sperm perforation. The pvm isolated from the largest follicles was incubated with ejaculated sperm in the presence (B) or absence (A) of apyrase (200 units/ml) for $10 \mathrm{~min}$. After the incubation, the pvm was spread onto a glass slide and observed in a light microscope. (C) The number of holes per $0.15 \mathrm{~mm}^{2}$ was calculated from Image J Software and is expressed as mean \pm s.D. The images shown in (A), (B) and (C) are representative of repeated experiments. Bar represents $50 \mu \mathrm{m}$.
(Perry \& Gilbert 1979). The ZP1 protein probably also passes through the gap between the granulosa cells, and the ubiquitination might take place during the transport. Although the possibility cannot be completely ruled out, the intracellular ubiquitination of ZP1 could not have occurred because the lysate obtained from granulosa and theca cells does not contain immunoreactive materials against the anti-ZP1 antibody (data not shown). Identifying the site of ubiquitination and the ubiquitination enzyme responsible for ZP1 modification remains to be achieved in a future study.

In our immunoelectron microscopy tests, immunoreactive proteasome localized in the acrosome of the sperm head. During the AR in quail, the acrosomal cap, which is $4 \mu \mathrm{m}$ in size, comes off from the head, and the acrosomal contents are thought to be released. Given this, the proteasome that is released from the sperm during the AR might degrade the ubiquitinated ZP1 in fertilization. In the mouse, Inoue et al. (2011) clearly demonstrated that the acrosome-reacted sperm recovered from the perivitelline space can penetrate the ZP again and can fertilize to produce healthy pups. In addition, Jin et al. (2011) recently showed evidence that most fertilizable sperm is not intact sperm but acrosomereacted sperm, which is revealed by live imaging of the sperm-egg interaction. From these observations, the acrosomal contents including protease are not that important for achieving fertilization in mice. In birds, the holes that form in the pvm due to sperm during fertilization are huge compared with those in the mammalian ZP, the fibers in the holes disappear, and the edges of the holes appear to be smooth (Takeuchi et al. 2001). The holes do not develop only by the mechanical force generated by sperm motility but also by other means, namely, a protease. We do not know whether acrosome-reacted sperm can hydrolyze pvm owing to the technical difficulty of collecting acrosomereacted sperm without any contamination by intact sperm; however, on the basis of the observations mentioned earlier, we believe that an acrosome-derived protease like proteasome is important for the hydrolysis of pvm in birds based on the observations mentioned earlier.

In western blot analysis of the cavitated sperm, the immunoreactive materials recognized by the anti-20S proteasome subunit were localized in both the cytosol and the membrane fractions. This result indicates the presence of membrane-associated proteasome in the sperm. Sawada et al. (2002b) also reported that sperm proteasome appears to be localized on the sperm surface because cell-impermeable NHS-LC-biotin is capable of labeling the sperm proteasome. More recently, Yokota et al. (2011) demonstrated that the 16 C-terminal residues of the sperm proteasome $\alpha 6$ subunit are posttranslationally and proteolytically processed in a testis-specific manner in $\mathrm{H}$. roretzi, and the possible involvement of this processing in the extracellular transport of the sperm 
proteasome has been suggested. Taken together with our experimental data, we think that the proteasome in quail sperm might localize not only inside the acrosomal matrix but also on the surface of the cells. The specific role of proteasome that might be exposed to the outside on the surface of the sperm remains to be studied.

In conclusion, we demonstrated for the first time that the ubiquitin-proteasome system functions in avian fertilization. The target protein for sperm proteasome was found to be ubiquitinated pvm ZP1, and the presence of the posttranslational ubiquitination of ZP1 during transport was suggested. Although the timing of the ubiquitination of the target protein differs in different species, the ubiquitin-proteasome system might be universally conserved in animal fertilization. The avian system is a beneficial model for studying the role of sperm proteasome in fertilization because avian sperm can produce a huge hole on the pvm, and large quantities of the target protein, ZP1, can be isolated from a large oocyte.

\section{Materials and Methods}

\section{Animals and tissue preparation}

Male and female Japanese quail, Coturnix japonica, 15-30 weeks of age (Kato-farm, Toyohashi, Japan), were maintained individually under a photoperiod of $14 \mathrm{~h}$ light: $10 \mathrm{~h}$ darkness (with the light on at 0500) and were provided with water and a commercial diet (Tokai-Hokuriku Nosan, Chita, Japan) ad libitum. The female animals were decapitated and the largest preovulatory follicles were dissected. The granulosa layer from the largest preovulatory follicles was isolated as a sheet of granulosa cells sandwiched between the pvm and basal laminae, as described previously (Gilbert et al. 1977). The pvm was isolated according to a procedure described by Sasanami et al. (2002). The isolated pvm was used for in vitro assay for sperm-egg interaction. A proctodeal gland secretion was obtained manually from male quail as meringue-like foam. This foam was then centrifuged at $10000 \mathrm{~g}$ for $10 \mathrm{~min}$, and the supernatants collected were stored as a proctodeal gland secretion at $-80^{\circ} \mathrm{C}$ until use. All experimental procedures for the care and use of animals in this study were approved by the Animal Care Committee of Shizuoka University (approval number, 22-12).

\section{Purification of serum and pvm ZP1}

The pvm lysate was separated by one-dimensional SDS-PAGE, performed as described by Laemmli (1970), under nonreducing conditions on $12 \%(\mathrm{w} / \mathrm{v})$ polyacrylamide separating gel. The samples $(750 \mu \mathrm{g}$ protein per gel) were applied to $5 \%(\mathrm{w} / \mathrm{v})$ stacking gel without a comb for lane casting. After electrophoresis, the gel was stained with Copper Stain (Bio-Rad Laboratories), and the $97 \mathrm{kDa}$ ZP1 band was excised. The proteins were eluted by incubating the gel slices with $0.1 \%$ $(\mathrm{w} / \mathrm{v})$ SDS buffered at $\mathrm{pH} 8.0$ with $100 \mathrm{mmol} / \mathrm{l}$ Tris- $\mathrm{HCl}$ overnight at $4{ }^{\circ} \mathrm{C}$ with constant shaking. The eluent was then extensively dialyzed against water, lyophilized, and dissolved in PBS. The protein concentration of the sample was measured using a BCA protein assay kit (Pierce, Rockford, IL, USA).

To prepare the affinity gel for the separation of serum ZP1, the IgG fractionated from anti-ZP1 antiserum (Kinoshita et al. 2008) with a HiTrap Protein A FF affinity column (Amersham Pharmacia Biotech) that had been covalently coupled to NHS-activated sepharose (Amersham Pharmacia Biotech) according to the manufacturer's instructions. The serum of laying birds was incubated with the affinity gel for $16 \mathrm{~h}$ at $4{ }^{\circ} \mathrm{C}$. After extensive washing with $\mathrm{PBS}$, the gel was eluted with elution buffer $\left(1 \mathrm{~mol} / \mathrm{l} \mathrm{CH}{ }_{3} \mathrm{COOH}, 0.1 \mathrm{mmol} / \mathrm{l}\right.$ glycine, $\left.\mathrm{pH} 2.5\right)$ and the eluent containing serum ZP1 was recovered. The $97 \mathrm{kDa}$ serum ZP1 was purified with the same procedure as that used to purify pvm ZP1. The purity of the ZP1s was confirmed by CBB staining after separation of the protein by SDS-PAGE.

\section{Semen collection and preparation}

Semen obtained from two to three males was suspended in Hanks' balanced salt solution (HBSS) containing $1.25 \mathrm{mmol} / \mathrm{l}$ of $\mathrm{CaCl}_{2}$ and $0.1 \%(\mathrm{v} / \mathrm{v})$ of proctodeal gland secretion. We added proctodeal gland secretion to the incubation mixture as it inhibits agglutination of the quail sperm. The concentrations of sperm were measured with a hemocytometer and the sperm viabilities were determined using the LIVE/DEAD sperm viability kit according to the manufacturer's instructions (Molecular Probes, Eugene, OR, USA). In all the experiments, sperm were incubated at $39^{\circ} \mathrm{C}$.

To obtain the sperm membrane or cytosol fraction, the ejaculates were washed three times with ice-cold PBS with repeated centrifugation at $800 \mathrm{~g}$ for $5 \mathrm{~min}$ at $4{ }^{\circ} \mathrm{C}$, and the final pellet was suspended in buffer containing $150 \mathrm{mmol} / \mathrm{l} \mathrm{NaCl}$ and $20 \mathrm{mmol} / \mathrm{l}$ HEPES (pH 7.4). The suspension was cavitated with a cell disruptor (Parr Instrument Company, Moline, IL, USA) at 400 psig, and the cellular debris was removed by centrifugation at $10000 \mathrm{~g}$ for $10 \mathrm{~min}$. The supernatants were further centrifuged at $100000 \mathrm{~g}$ for $30 \mathrm{~min}$, and the supernatants (cytosol) and the precipitates (membrane) stored at $-80^{\circ} \mathrm{C}$ until use.

\section{In vitro assay for sperm-egg interaction}

A piece of pvm, $\sim 8 \mathrm{~mm}$ in diameter, was incubated in a microtest tube with $0.5 \mathrm{ml}$ sperm suspension at $1 \times 10^{7} \mathrm{sperm} / \mathrm{ml}$ in HBSS at $39^{\circ} \mathrm{C}$ for $0-60 \mathrm{~min}$. After incubation, the reaction was terminated by placing the tube on ice and the pvm was washed three times with ice-cold PBS, then transferred onto a glass slide, and stained with Schiff's reagent after fixation with $3.7 \%$ $(\mathrm{v} / \mathrm{v})$ formaldehyde in PBS. The holes that formed on the pvm in the $\times 200$ field were photographed in a light microscope (BX 51, Olympus Optics, Tokyo, Japan), and the number of holes per $0.15 \mathrm{~mm}^{2}$ was calculated using Image J software (version 1.44). At least five areas were randomly selected for enumeration of perforations. For the analysis of the interaction of purified ZP1 with sperm, ejaculated sperm at $1 \times 10^{7}$ sperm $/ \mathrm{ml}$ in HBSS was mixed with equal amounts of the purified ZP1 solution diluted in HBSS $(50 \mu \mathrm{g} / \mathrm{ml})$ and incubated as described earlier. 
To observe the inhibitory activity of the anti-20S proteasome antibodies, the pvm was added into a tube containing either anti-20S proteasome antibody (anti- $\alpha 5$ or anti- $\alpha 7$ ) (Wakata et al. 2004) or normal guinea pig serum diluted 1:200 with HBSS, and sperm suspension was then added at $1 \times 10^{7} \mathrm{sperm} / \mathrm{ml}$. The effects of $50 \mu \mathrm{M}$ carbobenzoxyLeu-Leu-Leu-H (MG132, Sigma), a proteasome inhibitor, $50 \mu \mathrm{M}$ [2S-3S]-trans-epoxysuccinyl-L-leucylamido-3-methylbutane ethyl ester (E64d, Sigma), a cysteine protease inhibitor, or 200 units $/ \mathrm{ml}$ apyrase (Sigma) that catalyzes the hydrolysis of ATP and ADP on the sperm perforation of the pvm were also tested as described earlier. The concentration of MG132 and E64d was adopted from Yokota \& Sawada (2007b). Appropriate vehicle (dimethyl sulfoxide for MG132 and E54d, saline for apyrase) was included in the incubation mixture for the control experiments and the final concentration of each solvent was adjusted to $0.1 \%$. For evaluation of the acrosomal status of the sperm, the ejaculated spermatozoa were incubated with or without purified ZP1 for $10 \mathrm{~min}$ as described previously (Sasanami et al. 2007).

\section{Gel electrophoresis, western blot, and ligand blot analysis}

For western blotting, the cavitated sperm proteins separated by SDS-PAGE were electrotransferred to a PVDF membrane (Immobilon-P, Millipore, Bedford, MA, USA) (Matsudaira 1987). The membrane incubated with anti-20S proteasome antibody (anti- $\alpha 5$ or anti- $\alpha 7$ ) or normal guinea pig serum diluted 1:2000 followed by HRP-conjugated anti-guinea pig IgG (Cappel, Durham, NC, USA) as a secondary antibody was visualized by means of a chemiluminescent technique (Amersham Pharmacia Biotech). For detection of ubiquitinated ZP proteins, the membrane that immobilized the purified ZP1 or ZP3 was reacted with anti-ubiquitin antibody (P4D1, Santa Cruz Biotechnology, Santa Cruz, CA, USA), which reacts with free ubiquitin, poly-ubiquitinated, and mono-ubiquitinated proteins. The signals were detected with HRP-conjugated antimouse IgG (Cappel) as a secondary antibody. The immunoblot for ZP1 and ZP3 was performed as described previously (Sasanami et al. 2002, 2006). For ligand blot analysis, a PVDF strip electrotransferred with pvm lysate or purified ZP1 was incubated with $\left[{ }^{3} \mathrm{H}\right]$-labeled ZP3 as described previously (Ohtsuki et al. 2004).

\section{Immunoelectron microscopy}

Ejaculated sperm embedded in $3 \%(\mathrm{w} / \mathrm{v})$ agarose were fixed with $2.5 \%(\mathrm{v} / \mathrm{v})$ glutaraldehyde in $0.1 \mathrm{M}$ cacodylate buffer ( $\mathrm{pH}$ 7.4) overnight at $4{ }^{\circ} \mathrm{C}$. The specimens were embedded in Lowicryl $\mathrm{K}_{4} \mathrm{M}$ resin (Polysciences, Warrington, $\mathrm{PA}, \mathrm{USA}$ ). Thin sections were first treated with anti- $\alpha 5$ antibody $(1: 20)$ or anti- $\alpha 5$ antibody pre-absorbed with antigen protein $(1: 20)$ and then with a gold-conjugated goat antiserum against guinea pig IgG (1:10) (E-Y Laboratories, San Mateo, CA, USA). The sections were stained with uranyl acetate and observed with a model H-8000 electron microscope (Hitachi, Tokyo, Japan).

\section{Declaration of interest}

The authors declare that there is no conflict of interest that could be perceived as prejudicing the impartiality of the research reported.

\section{Funding}

This work was supported by financial support from a Grant-in-Aid for Scientific Research (B) (General) (24380153 to $T$ Sasanami), a Grant-in-Aid for Scientific Research on Innovative Areas (24112710 to T Sasanami), and the Sumitomo Foundation (101069 to T Sasanami).

\section{Acknowledgements}

The authors are grateful to Mr Y Ogasa, Dr K Yoshimatsu, and Miss M Matsuzaki for technical assistance.

\section{References}

Baba T, Azuma S, Kashiwabara S \& Toyoda Y 1994 Sperm from mice carrying a targeted mutation of the acrosin gene can penetrate the oocyte zona pellucida and effect fertilization. Journal of Biological Chemistry 269 31845-31849.

Bellairs R 1965 The relationship between oocyte and follicle in the hen's ovary as shown by electron microscopy. Journal of Embryology and Experimental Morphology 13 215-233.

Florman HM \& Ducibella T 2006 Fertilization in mammals. In Physiology of Reproduction, 3rd edn, vol 1, pp 55-112. Ed JD Neill. St Louis, MO: Elsevier Academic Press.

Gilbert AB, Evans AJ, Perry MM \& Davidson MH 1977 A method for separating the granulosa cells, the basal lamina and the theca of the preovulatory ovarian follicle of the domestic fowl (Gallus domesticus). Journal of Reproduction and Fertility 50 179-181. (doi:10.1530/jrf.0. 0500179)

Inoue N, Satouh Y, Ikawa M, Okabe M \& Yanagimachi R 2011 Acrosomereacted mouse spermatozoa recovered from the perivitelline space can fertilize other eggs. PNAS 108 20008-20011. (doi:10.1073/pnas. 1116965108)

Jin M, Fujikawa E, Kakiuchi Y, Okabe M, Satouh Y, Baba SA, Chiba K \& Hirohashi N 2011 Most fertilizing mouse spermatozoa begin their acrosome reaction before contact with the zona pellucida during in vitro fertilization. PNAS 108 4892-4896. (doi:10.1073/pnas.1018202108)

Kinoshita M, Mizui K, Ishiguro T, Ohtsuki M, Kansaku N, Ogawa $H$, Tsukada A, Sato T \& Sasanami T 2008 Incorporation of ZP1 into perivitelline membrane after in vivo treatment with exogenous ZP1 in Japanese quail (Coturnix japonica). FEBS Journal 275 3580-3589. (doi:10.1111/j.1742-4658.2008.06503.x)

Kong M, Diaz ES \& Morales P 2009 Participation of the human sperm proteasomes in the capacitation process and its regulation by protein kinase A and tyrosine kinase. Biology of Reproduction 80 1026-1035. (doi:10.1095/biolreprod.108.073924)

Laemmli UK 1970 Cleavage of structural proteins during the assembly of the head of bacteriophage T4. Nature 227 680-685. (doi:10.1038/ 227680a0)

Matsudaira P 1987 Sequence from picomole quantities of proteins electroblotted onto polyvinylidene difluoride membranes. Journal of Biological Chemistry 262 10035-10038.

McRorie RA \& Williams WL 1974 Biochemistry of mammalian fertilization. Annual Review of Biochemistry 43 777-803. (doi:10.1146/annurev.bi. 43.070174.004021)

Ohtsuki M, Hanafy AM, Mori M \& Sasanami T 2004 Involvement of interaction of ZP1 and ZPC in the formation of quail perivitelline membrane. Cell and Tissue Research 318 565-570. (doi:10.1007/ s00441-004-1000-9) 
Pasten C, Morales P \& Kong M 2005 Role of the sperm proteasomes during fertilization and gamete interaction in the mouse. Molecular Reproduction and Development 71 209-219. (doi:10.1002/mrd.20280)

Perry MM \& Gilbert AB 1979 Yolk transport in the ovarian follicle of the hen (Gallus domesticus): lipoprotein-like particles at the periphery on the oocyte in the rapid growth phase. Journal of Cell Science 39 257-272.

Richardson ME, Korn N, Bodine AB \& Thurston RJ 1992 Kinetic and inhibition studies with turkey acrosin. Poultry Science 71 1789-1793. (doi:10.3382/ps.0711789)

Robertson L, Wishart GJ \& Horrocks AJ 2000 Identification of perivitelline $\mathrm{N}$-linked glycans as mediators of sperm-egg interaction in chickens. Journal of Reproduction and Fertility 120 397-403. (doi:10.1530/reprod/ 120.2.397)

Sakai N, Sawada H \& Yokosawa H 2003 Extracellular ubiquitin system implicated in fertilization of the ascidian, Halocynthia roretzi: isolation and characterization. Developmental Biology 264 299-307. (doi:10.1016/j.ydbio.2003.07.016)

Sasanami T, Pan J, Doi Y, Hisada M, Kohsaka T \& Toriyama M 2002 Secretion of egg envelope protein ZPC after C-terminal proteolytic processing in quail granulosa cells. European Journal of Biochemistry 269 2223-2231. (doi:10.1046/j.1432-1033.2002.02880.x)

Sasanami T, Pan J \& Mori M 2003 Expression of perivitelline membrane glycoprotein ZP1 in the liver of Japanese quail (Coturnix japonica) after in vivo treatment with diethylstilbestrol. Journal of Steroid Biochemistry and Molecular Biology 84 109-116. (doi:10.1016/S0960-0760(03) 00008-6)

Sasanami T, Ohtsuki M, Ishiguro T, Matsushima K, Hiyama G, Kansaku N, Doi Y \& Mori M 2006 Zona pellucida domain of ZPB1 controls specific binding of ZPB1 and ZPC in Japanese quail (Coturnix japonica). Cells, Tissues, Organs 183 41-52. (doi:10.1159/000094905)

Sasanami T, Murata T, Ohtsuki M, Matsushima K, Hiyama G, Kansaku N \& Mori M 2007 Induction of sperm acrosome reaction by perivitelline glycoprotein ZP1 in Japanese quail (Coturnix japonica). Reproduction 133 41-49. (doi:10.1530/REP-06-0104)

Sasanami T, Yoshizaki N, Dohra H \& Kubo H 2011 Sperm acrosin is responsible for the sperm binding to the egg envelope during fertilization in Japanese quail (Coturnix japonica). Reproduction 142 267-276. (doi:10.1530/REP-11-0120)

Sawada H, Sakai N, Abe Y, Tanaka E, Takahashi Y, Fujino J, Kodama E, Takizawa S \& Yokosawa H 2002a Extracellular ubiquitination and proteasomes-mediated degradation of the ascidian sperm receptor. PNAS 99 1223-1228. (doi:10.1073/pnas.032389499)

Sawada H, Takahashi Y, Fujino J, Flores SY \& Yokosawa H 2002b Localization and roles in fertilization of sperm proteasomes in the ascidian Halocynthia roretzi. Molecular Reproduction and Development 62 271-276. (doi:10.1002/mrd.10089)

Slowinska M, Olczak M, Liszewska E, Watorek W \& Ciereszko A 2010 Isolation, characterization and cDNA sequencing of acrosin from turkey spermatozoa. Comparative Biochemistry and Physiology. Part B, Biochemistry \& Molecular Biology 157 127-136. (doi:10.1016/j.cbpb. 2010.05.011)

Sutovsky P, Manandhar G, Mccauley TC, Caamano JN, Sutovsky M, Thompson WE \& Day BN 2004 Proteasomal interference prevents zona pellucida penetration and fertilization in mammals. Biology of Reproduction 71 1625-1637. (doi:10.1095/biolreprod.104.032532)

Takeuchi Y, Cho R, Iwata Y, Nishimura K, Kato T, Aoki N, Kitajima K \& Matsuda T 2001 Morphological and biochemical changes of isolated chicken egg-envelope during sperm penetration: degradation of the 97-kilodalton glycoprotein is involved in sperm-driven hole formation on the egg-envelope. Biology of Reproduction 64 822-830. (doi:10.1095/ biolreprod64.3.822)

Wakata Y, Tokumoto M, Horiguchi R, Ishikawa K, Nagahama Y \& Tokumoto T 2004 Identification of $\alpha$-type subunits of the Xenopus $20 \mathrm{~S}$ proteasomes and analysis of their changes during the meiotic cell cycle. BMC Biochemistry 5 18. (doi:10.1186/1471-2091-5-18)

Wyburn GM, Aitken RNC \& Johnston HS 1965 The ultrastructure of the zona radiata of the ovarian follicle of the domestic fowl. Journal of Anatomy 99 469-484.

Yamagata K, Murayama M, Okabe M, Toshimori K, Nakanichi T, Kashiwabara S \& Baba T 1998 Acrosin accelerates the dispersal of sperm acrosomal proteins during acrosome reaction. Journal of Biological Chemistry 273 10470-10474. (doi:10.1074/jbc.273.17.10470)

Yokota N \& Sawada H 2007a Sperm proteasomes are responsible for the acrosome reaction and sperm penetration of the vitelline envelope during fertilization of the sea urchin Pseudocentrotus depressus. Developmental Biology 308 222-231. (doi:10.1016/j.ydbio. 2007.05.025)

Yokota N \& Sawada H 2007b Effects of proteasomes inhibitors on fertilization of the sea urchin Anthocidaris crassispina. Biological \& Pharmaceutical Bulletin 30 1332-1335. (doi:10.1248/bpb.30.1332)

Yokota N, Kataoka Y, Hashii N, Kawasaki N \& Sawada H 2011 Spermspecific $C$ terminal processing of the proteasomes PSMA1/ $\alpha 6$ subunit. Biochemical and Biophysical Research Communications 410 809-815. (doi:10.1016/j.bbrc.2011.06.069)

Zimmerman SW, Manandhar G, Yi Y, Gupta SK, Sutovsky M, Odhiambo JF, Powell D, Miller DJ \& Sutovsky P 2011 Sperm proteasomes degrade sperm receptor on the egg zona pellucida during mammalian fertilization. PLoS ONE 6 e17256. (doi:10.1371/journal.pone.0017256)

Received 4 May 2012

First decision 23 May 2012

Revised manuscript received 5 July 2012

Accepted 2 August 2012 